\title{
Event related potentials recorded in patients with locked-in syndrome
}

\author{
Marco Onofrj, Astrid Thomas, Cristina Paci, Michele Scesi, Renato Tombari
}

\begin{abstract}
Objective-To determine the possibility of recording "cognitive" event related potentials (ERPs) in locked-in patients and therefore to determine whether ERPs can have a role in differential diagnosis of coma.

Methods-ERPs to classic auditory or visual "odd ball paradigms" were recorded three to four days, seven to eight days, and 30 to 60 days after admission to the intensive care unit, in four patients affected by basilar artery thrombembolism resulting in locked-in syndrome. Two patients (one 32 year old man, one 31 year old woman) could move the eyes laterally and vertically spontaneously and on command. One patient (a 39 year old man) had a "one and half syndrome", one patient (a 40 year old woman) could only elevate the left eyelid and eye. Results were compared with data from 30 age matched controls. In the last recording session a letter recognition paradigm was applied, in which ERPs were produced by the identification of letters forming a word. Results were compared with five age matched controls. Brainstem lesions extending to the pontomesencephalic junction were found on MRI and CT.

Results-ERPs to the oddball paradigms were recorded in three patients in the first recording session, in all patients in the second recording session. Latency, amplitude, and topographic distribution of ERP components were inside normal limits. With the letter recognition paradigm the patients could emit a P3 component to correspond with target letters, with the same margin of error as controls.

Conclusion-It is possible to record ERPs in patients with locked-in syndrome shortly after the acute ischaemic lesion, and therefore to assess objectively cognitive activities. Furthermore the letter recognition paradigm could be implemented to facilitate linguistic communication with patients with locked-in syndrome.
\end{abstract}

(F Neurol Neurosurg Psychiatry 1997;63:759-764)

Keywords: locked-in syndrome; P3-P300; event related potentials; basilar artery thromboembolism

P3 (or P300) event related potentials (ERPs) are electric signals from the brain recorded during the performance of cognitive tasks. Several different paradigms have been devised to elicit the P3-ERP. These include active ${ }^{1}$ and passive ${ }^{2}$ discrimination between frequent or infrequent (rare) targets, recognition of known versus unknown faces, ${ }^{3}$ mental rotation task,${ }^{4}$ preparation for a motor task, ${ }^{5}$ and recognition of a missing stimulus inside a rhythmic sequence. ${ }^{6}$ P3 was described as an activity reflecting attention, ${ }^{7}$ context updating, ${ }^{8}$ decisional closure, ${ }^{9}$ or as a subcortical reafference subserving the build up of conscious perception. ${ }^{10}$

As P3 was recorded in patients with severe lesions of the temporal lobe and amnesia, ${ }^{11-14}$ the hypotheses suggesting that its origin is in the medial temporal lobe $e^{15}$ have been reconsidered: recent reports show that P3 arises almost simultaneously from multiple cortical areas, ${ }^{17-19}$ in agreement with the studies concluding that brainstem structures or the thalamus could be the trigger of $\mathrm{P} 3 .^{20-22}$ The studies quoted above suggest that $\mathrm{P} 3$ recordings could be applied in differential diagnosis of coma, such as catatonic stupor, locked-in syndrome, or diencephalic lesions: published papers on P3 ERPs and coma are, however, surprisingly few, ${ }^{23-25}$ and only one methodological study, performed in normal controls, hypothesised that P3 could be recorded in locked-in patients, and used to drive a computer based communication system. ${ }^{26}$

The present paper shows actual recordings of P3 ERPs in four patients with locked-in syndrome: P3-ERPs were recorded with classic oddball paradigms in the visual and the auditory modality (the patient had to recognise target stimuli). Furthermore, patients were presented a letter recognition task. The patients produced P3-ERPs to target letters, and a template was devised to identify P3 and therefore the selected letter.

\section{Case reports}

PATIENT 1

Patient 1 was a 32 year old man, a professional cook, $172 \mathrm{~cm}$ in height, $68 \mathrm{~kg}$ weight, a non-smoker, with a negative medical history. He arrived at the emergency unit of our hospital because of headache followed by "abnormal vision" and numbness of the right arm and leg. Blood pressure was $200 / 120 \mathrm{~mm} \mathrm{Hg}$ at admission. Within 12 hours he became stuporous, anartric, and tetraparetic with bilateral Babinski signs, he had two generalised tonic-clonic seizures, painful stimuli induced decerebrate posturing, and lateral eye movements. The first $\mathrm{CT}$ was reported as negative. $\mathrm{He}$ received 20000 units of heparin in 24 hours. He developed central hyperventilation (frequency 40-50/ min, $\mathrm{PO}_{2} 85-90 \mathrm{~mm} \mathrm{Hg}, \mathrm{PCO}_{2} 25-40 \mathrm{~mm} \mathrm{Hg}$ ). 
He was intubated and artificially ventilated and treated with $2 \mathrm{mg} / \mathrm{kg} / \mathrm{h}$ thiopental. His CSF was normal. Within three days it was possible to interrupt artificial ventilation and he underwent tracheotomy. A new CT showed a caudal pontine ischaemic lesion, prominent on the right side. The patient was then awake. Painful stimuli induced blinking and decerebrate posturing and he could blink and move the eyes laterally and vertically spontaneously and on command. $\mathrm{He}$ understood verbal commands and spasmodic laughing or crying was elicited by appropriate stimuli. An EEG showed posterior 9-10 Hz rhythms, disappearing on eye closure, and sporadic synchronous $5-6 \mathrm{~Hz}$ sequences lasting for one to two seconds. During sleep, slow activity, $\mathrm{K}$ complexes, spindles, and vertex waves were recorded. MRI showed a paramedian (right) area hypointense in T1 weighted images and hyperintense in T2 images (fig 1).

PATIENT 2

Patient 2 was a 39 year old man, a library clerk, $175 \mathrm{~cm}$ in height, $81 \mathrm{~kg}$ weight, previously affected by "basilar artery migraine". $\mathrm{He}$ arrived at the emergency unit of our hospital because of headache and right brachiocrural hemiparesis. Within six hours he became dysartric (nasal and scanned speech), the right hemiparesis subsided, and left faciobrachial paresis was seen. The first CT was reported as normal. The first EEG showed posterior $\alpha$ rhythms unresponsive to stimuli. $\mathrm{He}$ was treated with 20000 units of heparin intravenously, but he became tetraparetic and stuporous, with ataxic breathing. He was intubated and artificially ventilated; within five days it was possible to interrupt artificial ventilation and he underwent tracheotomy. The patient was then awake. Painful stimuli elicited blinking and decerebrate posturing, he could move his eyes spontaneously and on command vertically and to the left with a contralateral "one and a half" syndrome. He laughed and cried spasmodically. A second CT showed a caudalpontine ischaemic lesion. An EEG showed posterior $10-11 \mathrm{~Hz}$ rhythms, disappearing when his eyes were closed. Slow activity, K complex, spindles, vertex V waves, and REM desynchronisation were seen during sleep. Brain MRI confirmed the paramedian area, which was hypointense in T1 weighted images and hyperintense in T2 images (fig 1).

\section{PATIENT 3}

Patient 3 was a 40 year old woman, a housewife, obese $(163 \mathrm{~cm}$ in height, $91 \mathrm{~kg}$ weight), with a negative medical history. She was admitted to the surgery of our hospital because of acute abdominal pain, ileus, and suspected mesenteric artery thrombosis. She became stuporous and tetraparetic, with ataxic
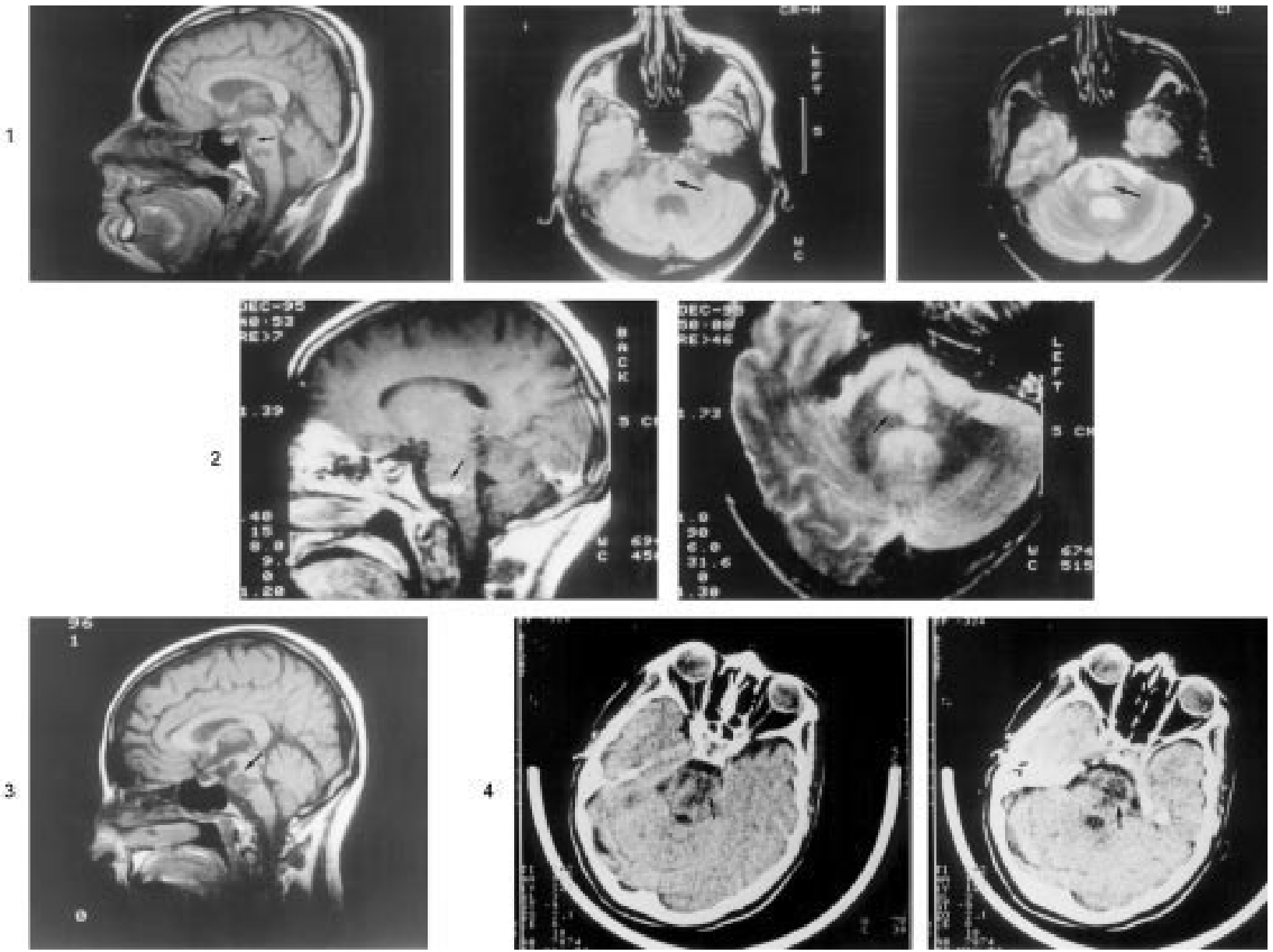

Figure 1 MRI (patients 1,2, and 3) and CT (patient 4) showing brain stem lesions in four patients with locked-in syndrome. (1) MRI, T1 time echo (sagittal and first axial), T2 time echo (last axial) of patient 1. (2) MRI, T2 time echo (sagittal and axial) of patient 2. (3) Sagittal MRI, T1 time echo of patient 3. (4) CT showing pontine hypointensities in patient 4. Arrows point to lesions. 
breathing, and underwent tracheotomy and artificial ventilation. An EEG showed posterior $\alpha$ rhythms, unmodified by stimuli. The first CT showed hypointensity in pontine structures; haematological studies showed platelet decrement and increment of fibrinogen degradation products. She was treated with 20000 units heparin daily.

Within three days she was awake. She could elevate the left eyelid and the left eye spontaneously and on command and EEG showed 10-11 Hz $\alpha$ rhythms and normal sleep patterns during sleep. Painful stimuli elicited spasmodic crying and decerebrate posturing. She developed left femoral vein thrombosis. A new CT showed the hypointense lesions of the pons, and repeated MRI (fig 1) confirmed earlier findings. She was found positive for antiphospholipid antibodies.

PATIENT 4

Patient 4 was a 31 year old housewife, normotype $(166 \mathrm{~cm}$ in height, $56 \mathrm{~kg}$ weight). She underwent a caesarean section due to initial gestosis, with delivery of a normal baby. She was somnolent-stuporous for three days and she was then transferred from the obstetric division of a nearby hospital to our department. At the first neurological examination she was found to be tetraplegic, but in a waking state. She was able to blink and move her eyes vertically, spontaneously and on command. Brain CT showed hypointensity of the caudal pons, left hypointensity of the rostral pons, and bilateral hypointensities of the cerebral peduncles (fig 1). The first EEG showed 9-11 Hz $\alpha$ rhythmic activity, disappearing when her eyes closed, and normal sleep patterns. She was intubated and artificially ventilated for six days. Thoracic expansion than returned to normal. Ten days after admission to our department she had left hemiplegia, right hemiparesis (she could lift her left arm), facial diplegia, and tongue protrusion. Three months after rehabilitation therapy she could walk with the aid of a frame.

\section{Methods}

ERPs to the auditory and visual oddball paradigms were recorded three to four days after admission to the intensive care unit of our University, when the patients were artificially ventilated, seven to eight days later, when artificial ventilation could be discontinued and patients could be transferred to our neurology department, and 30 to 60 days later when patients were transferred to a rehabilitation unit. The letter recognition paradigm was used only in the last recording session. The ERPs were recorded according to methods described in detail elsewhere ${ }^{121922} 27$ from 21 scalp leads (comprising Fp, Fp2, Fpz, F7, F3, F4, F8, Fz, T7, C3, C4, T8, Cz, P7, P3, P4, P8, Pz, O1, $\mathrm{O} 2, \mathrm{Oz}$ ) with $\mathrm{Ag} / \mathrm{AgCl}$ cups, and four supplementary leads monitoring eye artefacts, all referenced to the linked earlobes with an interposite $10 \mathrm{kohm}$ resistance. Band pass filters were $0.1-100 \mathrm{~Hz}$, and dwell time was 0.4 $\mathrm{ms}$. In the binaural tones (plateau $100 \mathrm{~ms}$, risefall time $150 \mathrm{~ms}$, intensity $80 \mathrm{~dB}$ ) non-target (frequent) stimuli were $2000 \mathrm{~Hz}$ pure tones (plateau $100 \mathrm{~ms}$, rise-fall time $150 \mathrm{~ms}$, intensity $80 \mathrm{~dB}$ ) delivered with a random sequence and a minimum intersimulus interval of $1010 \mathrm{~ms}$. The frequent/rare probability ratio was $8 / 1$. The patients were instructed to count mentally rare stimuli and to communicate with eye blinks whether they understood the commands. In the visual odd ball paradigm, patients were asked whether they could identify vertical and horizontal bars subtending 30' of visual arc, $75 \%$ contrast presented on a pattern generator for $80 \mathrm{~ms}$, followed by an isoluminance screen $\left(75 \mathrm{~cd} / \mathrm{m}^{2}\right)$. The pattern generator subtended $25^{\circ}$ of visual angle and was placed at $1 \mathrm{~m}$ from the patient's head. The scleral reflection of the patterned stimuli was centred on the pupil. During the visual oddball paradigm patients had to recognise only horizontal target stimuli and communicated with eyeblinks whether they understood the task. Target and non-target stimuli were presented in a random sequence with a minimum interstimulus interval of $2010 \mathrm{~ms}$ and with a probability ratio of $1 / 8$. At the end of each recording session the patients blinked for as many times as many target stimuli were delivered. The latencies and amplitudes of ERPs recorded in these patients were compared with results obtained in two groups of 15 age matched controls (25 and 35 years old).

The letter recognition paradigm was developed as a simplification of the "mental prosthesis" paradigm introduced by Farwell and Donchin. ${ }^{26}$ Stimuli were grey letters subtending $5^{\circ}$ visual angles, presented on the same pattern generator screen used for the orientation "odd ball paradigm". Luminance of the blank screen and the screen surrounding the letters was $75 \mathrm{~cd} / \mathrm{m}^{2}$, luminance of the letters was $32 \mathrm{~cd} / \mathrm{m}^{2}$. Letters of the alphabet were presented randomly for one second on the screen. In the first recording session (template definition) five healthy controls, 30-43 years old, were asked to discriminate between letters forming the word "video" and other letters; the target letters were to be recognised only when presented in the appropriated sequence. The EEG was recorded from 15 scalp derivations (Fp1-2, F7-8, and T3-4 were excluded, one EOG channel was included), with $0.5-70 \mathrm{~Hz}$ band pass filtered and at a $1000 \mathrm{~Hz}$ sampling rate. Data were stored on the computer hard disk. Responses to target and non-target stimuli were averaged off line. The averaged response to target stimuli was used to define "template" characteristics, with the ESAOTE shape detection program, based on a time window (290-500 ms), slope of the descending $(0.15-0.4 \mathrm{mV} / \mathrm{ms})$ and ascending limb $(0.1-0.3$ $\mathrm{mV} / \mathrm{ms}$ ), and minimum duration of descending-ascending positive deflection (70$110 \mathrm{~ms}$ ).

In the second recording session each control was asked to select letters forming a word from the letter sequence presented on the screen, in five sets of six minutes each. The P3 template obtained in the first recording session in each control was used to compute the covariance of single responses to letter presentation with this 
Latencies and amplitudes of ERP components to the auditory and visual odd ball paradigm in patients and controls: measurements from $\mathrm{Pz}$ derivations

\begin{tabular}{|c|c|c|c|c|}
\hline & N1 & $P 2$ & N2 & P3 \\
\hline \multicolumn{5}{|c|}{ Auditory ERPS } \\
\hline \\
\hline Controls & $130.2(10.8)$ & $181.8(16.4)$ & $258.4(14.2)$ & $330.4(18.4)$ \\
\hline \multicolumn{5}{|l|}{ Patients: } \\
\hline 1 & 121 & 190 & 254 & 315 \\
\hline 2 & 148 & 202 & 265 & 335 \\
\hline 3 & 152 & 207 & 270 & 330 \\
\hline 4 & 132 & 188 & 260 & 325 \\
\hline \multicolumn{5}{|l|}{ Amplitude $(\mu \mathrm{V})$ : } \\
\hline Controls & $11.1(2.8)$ & $8.7(2.1)$ & $2.5(1.9)$ & $10.5(2.8)$ \\
\hline \multicolumn{5}{|l|}{ Patients: } \\
\hline 1 & 18.1 & 5.6 & 2.5 & 9.0 \\
\hline 2 & 15.5 & 7.2 & 4.1 & 10.8 \\
\hline 3 & 14.2 & 7.8 & 3.6 & 6.8 \\
\hline \multirow[t]{2}{*}{4} & 11.0 & 6.5 & 7.3 & 11.2 \\
\hline & \multicolumn{3}{|c|}{ Visual ERPS } & \\
\hline Latency (ms): & & & & \\
\hline Controls & $142.1(10.8)$ & $196.1(15.8)$ & $264.6(16.7)$ & $355.8(19.1)$ \\
\hline \multicolumn{5}{|l|}{ Patients: } \\
\hline 1 & 147 & 200 & 290 & 370 \\
\hline 2 & 132 & 188 & 274 & 338 \\
\hline 3 & 150 & 196 & 278 & 340 \\
\hline 4 & 145 & 202 & 269 & 330 \\
\hline \multicolumn{5}{|l|}{ Amplitude $(\mu \mathrm{V})$ : } \\
\hline Controls & $10.6(3.1)$ & $7.8(2.4)$ & $6.1(2.7)$ & $10.6(2.7)$ \\
\hline \multicolumn{5}{|l|}{ Patients: } \\
\hline 1 & 7.6 & 5.8 & 5.6 & 9.5 \\
\hline 2 & 6.8 & 4.8 & 6.7 & 11.3 \\
\hline 3 & 11.5 & 7.5 & 7.5 & 8.6 \\
\hline 4 & 9.3 & 9.9 & 4.8 & 12.8 \\
\hline
\end{tabular}

The mean values of controls included both age matched groups. Values in parentheses are SD.

template. The values obtained from this analysis were then used to determine the letter on which the subject was focusing attention. This same letter recognition paradigm was used in the last recording session. All four patients were initially asked to blink or elevate the eyelid when they understood the task. The letter sequences with the predefined target ("video") were then presented for 15 minutes and the template was obtained for each patient. In the second part of the paradigm 370-600 acquisitions were stored on the hard disk in 30 minutes and compared with the template. Attended letters were 45-72.

\section{Results}

Figure 1 shows the ischaemic lesion involving the caudal pontine area, prominent on the right.

The ERPs to the auditory and visual oddball paradigms were recorded in the first session in patients 2, 3, and 4 and only in the second and third session in patient 1 . They consisted of a "normal" N1-P1 sequence to frequent nontarget stimuli, and N, P1, N2, and P3 components to target stimuli (fig 2). Latencies, amplitudes, and scalp distributions of ERP components were within normal limits. The table reports mean values of age matched controls and results obtained in each patient. Significance probability mapping with $\mathrm{Z}$ transform evaluation ${ }^{28}$ was performed as a comparison between age matched maps and maps of each patient, and did not show any $\mathrm{Z}$ value above or below 2 SD in any of the scalp derivations. Paired Wilcoxon tests of N2 and P3 amplitude or latencies did not show any significant increment or decrement in the three recording sessions for patients 2,3 , and 4 and in the two recording sessions for patient 1 .

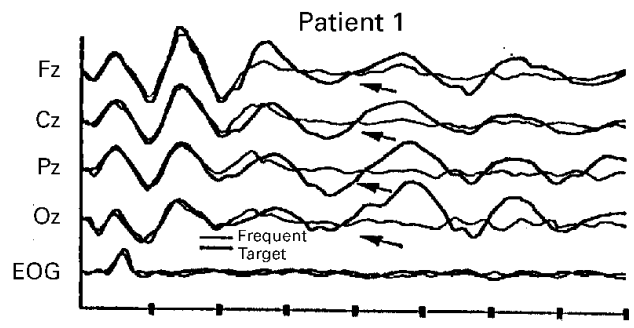

Patient 2

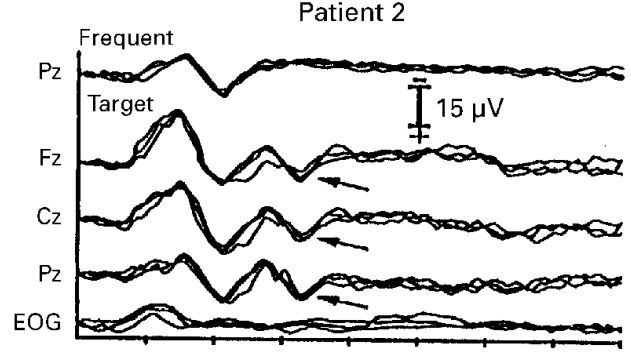

Patient 3

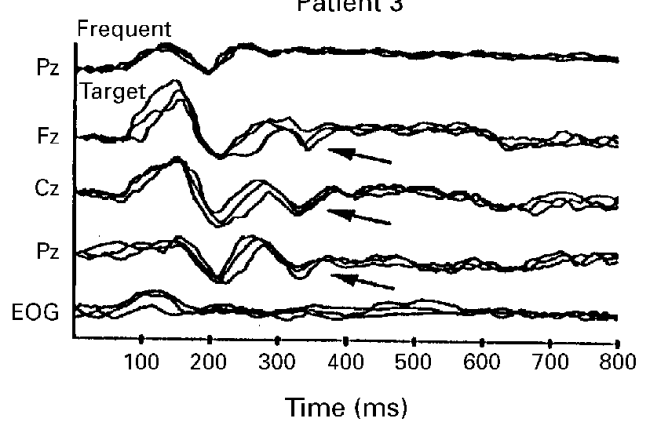

Figure 2 Visual oddball ERPs of patient 1, auditory oddball ERPs of patients 2 and 3. Arrows point to P3 ERP component. In patient 1 thick lines correspond to target stimuli ERPs, thin lines correspond to frequent (non-target) stimuli. In patients 2 and 3 the overlapping sets of traces show responses obtained in the three different recording sessions.

Test-retest reliability of P3 in patients with locked-in syndrome was $0.81-0.88$, not different from controls (0.81-0.91). Figure 2 shows ERPs recorded with the visual or auditory oddball paradigm in the first three patients.

The letter recognition task elicited positive P3 components to attended letters in all patients and controls. The averaged responses obtained during the template definition task consisted of $\mathrm{N} 1$ components recorded from $\mathrm{Cz}, \mathrm{Pz}, \mathrm{P} 3, \mathrm{P} 4, \mathrm{O} 1, \mathrm{O} 2$, and $\mathrm{Oz}$ leads to attended and unattended letters. N1 peaked at 120-185 ms, the amplitude (measured from $\mathrm{Pz}$ ) was 12.2 (SD 3.6) $\mathrm{mV}$ in patients and controls. A following peak, P2 was recorded also from central and frontal derivations, latency was from 182 to $260 \mathrm{~ms}$. Only attended letters elicited N2 (272-336 ms, amplitude 4.2 (SD 2.8) $\mathrm{mV}$ from $\mathrm{Pz}$ ) and $\mathrm{P} 3$ on $\mathrm{Cz}, \mathrm{C} 3, \mathrm{C} 4, \mathrm{P} 3$, $\mathrm{Pz}, \mathrm{P} 4, \mathrm{O} 1, \mathrm{Oz}$, and $\mathrm{O} 2$ leads (348-452 ms). The mean P3 latency was 408 (SD 18.5) $\mathrm{ms}$ in controls , 411 (SD 17.2) $\mathrm{ms}$ in the four patients. The mean amplitude from baseline, recorded from $\mathrm{Pz}$, was 7.7 (SD 2.1) $\mathrm{mV}$ in controls and 8.1 (SD 3.00) $\mathrm{mV}$ in patients. A Student's $t$ test comparison with the five controls was not significant. The template (shape detection program) obtained from 


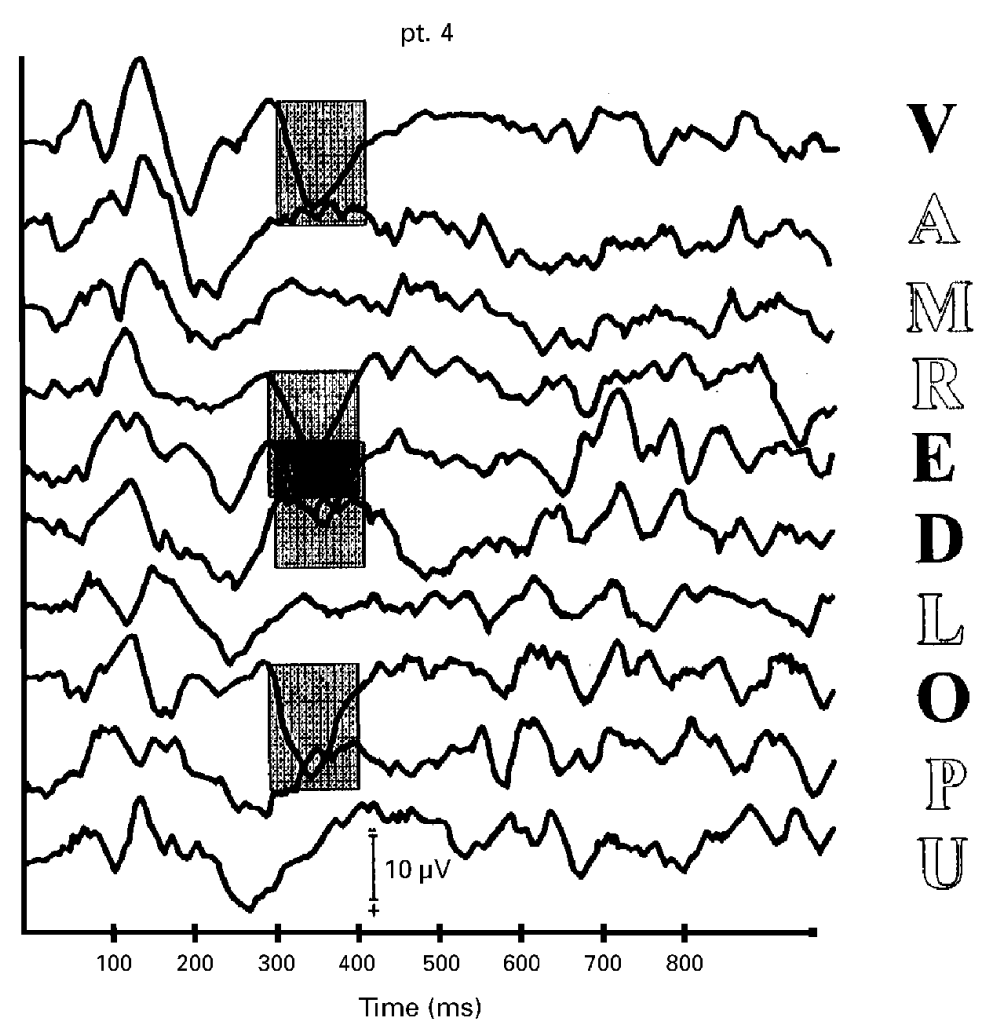

Figure 3 ERPs to the letter recognition paradigm in patient 4 . The sequence of traces from top to bottom show responses to single stimuli recorded from $\mathrm{Pz}$ derivation. The shaded frames represent the template identifying the corresponding P3 component. Attended letters are represented as dark; bright letters are unattended. The patient was emitting P3 ERPs to a sequence of four letters meaning "I see" in italian (VEDO).

averaged responses to attended letters could detect $67 \%-100 \%$ of $\mathrm{P} 3$ to attended letters in the rerun of the 30 minute acquisition during the second part of the paradigm. False positives were $10 \%-28 \%$ of detected P3s. Sequences of letters forming words could be detected with a $9 \%-43 \%$ margin of error (false positives, false negatives), single words ("hunger", "tired", "stupid" in italian) could be identified every 1.5-3.5 min. A $\chi^{2}$ comparison of detection errors did not show differences between patients and controls. Figure 3 shows a sequence of single responses obtained in patient 4 during the second part of the letter recognition paradigm.

\section{Discussion}

Although many studies ${ }^{29-31}$ have been published on the electrophysiological assessment of the locked-in syndrome, ERP recordings have never been described before. In our paper we show that ERP components could be detected in all four locked-in patients, with three different cognitive paradigms. In patient 1 , ERPs could not be recorded in the first recording session, probably because the initial reversible ischaemic lesion was extending rostrally to the pons.

As ERPs were recorded also during artificial ventilation in at least three patients, we suggest that the recording of ERP to the simple classic auditory oddball paradigm could become a useful tool in the evaluation of locked-in syndrome. Compared with the heterogeneity of findings with other electrophysiological techniques $^{29-31}$ that could only show normal or abnormal afference of sensory pathways, ERPs can evidence an ongoing cognitive activity, and thus confirm the integrity of some brain functions. The utility of ERP recording might seem overstated if only classic descriptions of locked-in syndrome are considered ${ }^{32}$ - that is, with integrity of ocular movements-thus implying that patients can communicate with eye movements. As we report in our description, however, eye movements can be severely altered in patients who are otherwise capable of complex cognitive activities (patient 2 had a one and half syndrome, patient 3 could only elevate one eye and one eyelid), and previous literature already described cases of complete ("mesencephalic") locked-in syndrome ${ }^{33}$ in patients incapable of eye movements. The involvement of eye and lid movements in our patients prompted the second part of our study, based on the letter recognition paradigm. In two of our patients the "augmentive communication systems" such as the ETRAN board or the words + infrared switch, that were developed for patients with amyotrophic lateral sclerosis $^{34}$ could not be used because of impairment of eye movement. We therefore tested the feasibility of the mental prosthesis project proposed by Farwell and Donchin. ${ }^{26}$ We showed that ERPs to a letter recognition paradigm can be recorded in patients with locked-in syndrome; and therefore that the mental prosthesis project might be feasible. Obviously the method used in our patients cannot have any practical application, because attended letters can be related to ERPs only after the acquired EEG activity is analysed off line with the template shape detection program, but further developments of on line EEG analysis systems might be able to overcome the technical problems.

A final comment relates to some of the current hypotheses on $\mathrm{P} 3$ generators. As reported in the introduction, some authors think that a trigger of $\mathrm{P} 3$ activity might be located in the thalamus or in brain stem structures. ${ }^{9} 1020-22$ One of the generators might correspond to the cholinergic neurons of the tegmentopontine area that constitute the trigger of widespread electrocortical activities. ${ }^{35}$ In our patients we showed, however, that ischaemic lesions were extended to the tegmental pons (fig 1): as P3 had normal latencies and amplitudes in these patients, we suggest that a P3 generator located in tegmentopontine areas is unlikely and, therefore, that any P3 generator must be located more rostrally.

1 Ritter W, Vaughan HG. Average evoked responses in vigilance and discrimination: a reassessment. Science 1969;164:326-8.

2 Polich J. P300 from a passive auditory paradigm. Electroencephalogr Clin Neurophysiol 1989;74:312-20.

3 Renault B, Signoret JL, Debuille B, Breton F, Bolgert F. Brain potentials reveal covert facial recognition in prosopagnosia. Neuropsychologia 1989;27:905-12.

4 Stuss DT, Sarazin FF, Leech EE, Picton TW. Event related potentials during naming and mental rotation. Electroencephalogr Clin Neurophysiol 1983;56:133-46.

5 Deecke L, Bashore T, Brunia CHM, Grunewald-Zuberbier E, Grunewald G, Kristeva R. Movement-associated potentials and motor control. (Epic 6) Ann NY Acad Sci 1984;425:398-428. 
6 Simson R, Vaughan HG, Ritter W. The scalp topography of potentials associated with missing visual or auditory stimuli.

7 Hillyard SA, Hink RF, Schwent VL, Picton TW. Electrical signs of selective attention in the human brain. Science

8 Donchin E, Coles MGH. Precommentary: is the P300 component a manifestation of contex updating? Behav Brain Sci 1988;11:355-425

9 Desmedt JE, P300 in serial tasks: an essential post-decision closure mechanism. Prog Brain Res 1981;54:682-6.

10 Picton TW. The P300 wave of human event-related potential. 7 Clin Neurophysiol 1992;9:456-79.

11 Rugg MD, Pickles CD, Potter DD, Roberts RC. Normal P300 following extensive damage to the left medial temporal lobe. F Neurol Neurosurg Psychiatry 1991;54:217-22.

12 Onofrj M, Fulgente T, Nobilio D, et al. P3 recordings in patients with bilateral temporal lobe lesions. Neurology patients with bila

13 Polich J, Squire LR. P300 from amnesic patients with bilateral hippocampal lesions. Electroencephalogr Clin Neuroeral hippocampal lesions.

14 O'Donnel BF, Cohen RA, Hokama H, et al. Electrical source analysis of auditory ERPs in medial temporal lobe amnestic syndrome. Electroencephalogr Clin Neurophysio 1993;87:394-402.

15 Wood CC, Allison T, Goff WR, Williamson PD, Spencer DB. On the neural origin of P300 in man. Progress in brain research. In: Kornhuber HH, Deecke L, eds. Amsterdam: Elsevier, 1980:51-66.

16 Tarkka IM, Stokic DS, Basile LFH, Papanicolaou AC. Electric source localization of the auditory P300 agrees with magnetic source location. Electroencephalogr Clin Neurophysiol 1995;96:538-45.

17 Halgren E, Baudena P, Clark JM, et al. Intracerebral potentials to rare target and distractor auditory and visual stimuli. I. Superior temporal plane and parietal lobe. Electroencephalogr Clin Neurophysiol 1995;94:191-220.

18 Baudena P, Halgren E, Heit G, Clarke JM. Intracerebral potentials to rare target and distractor auditory and visual potentials to rare target and distractor auditory and visual
stimuli. III. Frontal cortex. Electroencephal Clin Neurophysiol 1995;94:251-64.

19 Onofrj M, Fulgente T, Thomas A, Locatelli T, Comi G. P300 asymmetries in focal brain lesions are reference dependent. Electroencephalogr Clin Neurophysiol 1995;94 $432-9$

20 Yingling CD, Hosobuchi Y. A subcortical correlate of P300 in man. Electroencephal Clin Neurophysiol 1984;59:72-6.
21 Rogers RL, Baumann SB, Papanicolau AC. Localization of the P3 sources using magnetoencephalography and magnetic resonance imagin

22 Onofrj M, Curatola L, Malatesta G, et al. Delayed P3 event related potential (ERPs) in thalamic hemorrhage. Electroencephalogr Clin Neurophysiol 1992;83:52-61.

23 Yingling CD, Hosobuchi Y, Harrington M. P300 as a predictor of recovery from coma [letter]. Lancet 1990;336: 873.

24 Kane NM, Curry DH, Butler SR, Cummins BH. Electrophysiological indicator of awakening from coma [letter]. Lancet 1993;341:688.

25 Signorino M, D'Acunto S, Cercaci S, Pietropaoli P, Angeleri $\mathrm{F}$. The P300 in traumatic coma: conditioning of the odd-ball paradigm. Fournal of Psychophysiology 1997;11:5970 .

26 Farwell A, Donchin E. Talking off the top of the your head: toward a mental prosthesis utilizing event related brain potentials. Electroencephal Clin Neurophysiol 1988;70:510 23.

27 Onofrj M, Fulgente T, Thomas A. Event related potentials recorded in dorsal simultanagnosia. Cogn Brain Res 1995b; 3:25-32.

28 Duffy FH, Bartels PH, Bruchfiel JL. Significance probability mapping: an aid in the topographic analyses of brain electrical activity. Electroencephalogr Clin Neurophysiol 1981; 51:455-62.

29 Verhagen WIM, Huygen PLM, Schulte BPM. Clinical and electrophysiological study in a patient surviving from locked-in syndrome. Clin Neurol Neurosurg 1989;88:57-61.

30 Towle VL, Maselli R, Berstein LP, Spire J-P. Electrophysiologic studies on locked-in patients: heterogenity of findings. Electroencephalogr Clin Neurophysiol 1989;73:41926.

31 Guetling E, Isenmann S, Wichmann W. Electrophysiology in the locked-in-syndrome. Neurology 1996;46:1092-101.

32 Plum F, Posner JB. Diagnosis of stupor and coma. 3rd ed. Philadelphia: FA Davis, 1980.

33 Maurri S, Lambruschini I, Barontini F. Total mesencephalic "locked-in" syndrome. A case report and review of the lit"locked-in" syndrome. A case report
erature. Rev Neurol 1989;59:211-6.

34 Woltosz W. Personal computers as augmentative communication aids. Communication Outlook 1984;5:4-7.

35 Steriade M, Gloor P, Llinas RR, Lopes da Silva FH, Mesulam MM. Basic mechanisms of cerebral rhythmic activities. Electroencephalogr Clin Neurophysiol 1990;76:481-508. 